\title{
Impact of a randomized weight loss trial on breast tissue markers in breast cancer survivors
}

\author{
Christina M. Dieli-Conwright $\mathbb{D}^{1 凶}{ }^{凶}$, Maura Harrigan ${ }^{2}$, Brenda Cartmel ${ }^{2,3}$, Anees Chagpar ${ }^{3,4}$, Yalai Bai ${ }^{4}$, Fang-yong Li $\mathbb{i D}^{5}$, \\ David L. Rimm $\mathbb{1}^{3,4}$, Lajos Pusztai $\mathbb{D}^{3,4}$, Lingeng Lu $\mathbb{D}^{2}$, Tara Sanft ${ }^{3,4}$ and Melinda L. Irwin ${ }^{2,3}$
}

Few trials have examined the effect of lifestyle behavioral interventions on tissue markers in patients with cancer. The purpose of this study was to examine the feasibility and impact of a 6-month weight loss intervention on breast tissue and serum biomarkers in women with breast cancer. Fifty-one women who completed breast cancer treatment and had a BMl $\geq 25.0 \mathrm{~kg} / \mathrm{m}^{2}$ were randomized to a weight loss intervention or usual care. Breast tissue biopsies, fasting blood draw and body composition were collected at baseline and 6 months, with between-group changes examined using analysis of covariance method. Baseline and post-intervention biopsies were conducted in 49 and 42 women, respectively, with pre- and post-epithelial tissue available from 25 tissue samples. Average 6-month weight loss was $6.7 \%$ for the weight loss group and $2.0 \%$ increase for the usual care group ( $p<$ $0.0001)$. At baseline, body fat and serum insulin levels were inversely associated with breast tissue insulin receptor levels and CD68 $(p<0.05)$. At 6 months, favorable changes were observed in serum leptin and adiponectin levels and tissue CD163 among women randomized to weight loss vs. adverse change in women randomized to usual care $(p<0.05)$. Breast tissue biopsies are feasible to collect in a clinical research setting among breast cancer survivors, with weight loss favorably impacting metabolic and inflammatory markers associated with breast cancer.

npj Breast Cancer (2022)8:29; https://doi.org/10.1038/s41523-022-00396-z

\section{INTRODUCTION}

Obesity is a risk factor for 13 different cancers ${ }^{1}$, with mounting evidence that it may additionally play a role in mortality from breast cancer $^{2}$. The American Cancer Society recommends cancer survivors achieve and maintain healthy body weight, avoid physical inactivity, participate in 150 min of aerobic exercise plus two weekly sessions of strength training, and adhere to a dietary pattern high in vegetables, fruits, and whole grains ${ }^{3}$. Unfortunately, over $65 \%$ of breast cancer survivors are overweight or obese, with fewer than $30 \%$ engaging in recommended levels of physical activity ${ }^{4,5}$.

Possible mechanisms through which obesity affects breast cancer risk and mortality include inflammatory, immune, metabolic, and hormonal pathways ${ }^{6,7}$. A growing number of studies have examined serum biomarkers such as adiponectin, insulin, C-reactive protein, IL6 , TNF-a, and particularly leptin as said biomarkers are associated with these mechanisms in exercise and/or weight loss trials in cancer survivors or those at risk of developing cancer $^{8-12}$.

However, examining changes in tissue markers provides a more direct investigation of the impact of lifestyle interventions on tumor biology and allows for the assessment of underlying mechanistic pathways that cannot be examined from blood-based biomarkers alone. Preliminary evidence suggests that weight loss and/or exercise interventions may impact tumor biology in both breast cancer patients $\mathrm{s}^{9,10}$ and in women at increased breast cancer risk due to high body mass index (Table 1$)^{8}$. Said tissue biomarkers include tumor biomarkers (e.g., Ki67), inflammatory macrophage biomarkers of the $\mathrm{M} 1$ and $\mathrm{M} 2$ phenotype (e.g., CD163 and CD68), and biomarkers related to insulin (e.g., insulin receptor) strongly associated with recurrent breast cancer ${ }^{13}$.

Our previous Lifestyle, Exercise, and Nutrition (LEAN) trial demonstrated that a 6-month weight loss intervention was effective in producing an average $6 \%$ weight loss and $30 \%$ decrease in serum C-reactive protein levels among 100 breast cancer survivors who completed chemotherapy and/or radiation therapy ${ }^{14}$. Given these findings and given the paucity of research examining lifestyle interventions on tissue markers, we sought to determine the feasibility of collecting breast tissue via a baselineand 6-month biopsy among women treated for breast cancer, and to examine the effect of the LEAN intervention on changes in body composition and breast tissue and serum biomarkers, and explore associations between 6-month changes in body composition and serum and breast tissue biomarkers.

\section{RESULTS}

\section{Recruitment and feasibility of breast biopsy}

Between 1 February 2014 and 1 January 2016, 150 women were approached to participate in the LEAN biopsy study, with 110 women screened for participation in the trial. Fifty-one women of those screened (46\%) agreed to have two breast biopsies and complete all other baseline and 6-month measures; 26 were randomized to the intervention group, and 25 were randomized to the wait-list/usual care group. Baseline and post-intervention biopsies were conducted in 49 and 42 women, respectively, with pre- and post-epithelial tissue available from 25 women (Fig. 1). Of 91 biopsies conducted, there was one adverse event related to the biopsy. At a routine oncology care clinic visit 3 weeks after the baseline biopsy, a participant reported finding a lump in her breast. After conducting a physical exam, a mammogram, and an ultrasound, the lump was determined to be a small hematoma which may have been a result of the study biopsy. The participant continued in the study, including the 6-month breast biopsy.

\footnotetext{
${ }^{1}$ Division of Population Sciences, Department of Medical Oncology, Dana-Farber Cancer Institute, Harvard Medical School, Boston, MA, USA. ${ }^{2}$ Yale School of Public Health, New Haven, CT, USA. ${ }^{3}$ Yale Cancer Center, New Haven, CT, USA. ${ }^{4}$ Yale School of Medicine, New Haven, CT, USA. ${ }^{5}$ Yale Center for Analytic Sciences, New Haven, CT, USA.

凶email: ChristinaM_Dieli-Conwright@DFCI.Harvard.edu
} 


\section{Baseline characteristics}

Baseline characteristics were similar for women randomized to intervention and usual care (Table 2), except for body weight and BMI which were significantly higher in the usual care group whereby the women in the usual care group more likely to be obese than the intervention group $(p=0.002)$. The mean (SD) age of participants was 56.8 (8.9) years, and their mean BMI (SD) at enrollment was $32.8(6.0) \mathrm{kg} / \mathrm{m}^{2}$. They have diagnosed a mean of 3.3 years prior, and $54 \%$ presented with Stage I breast cancer.

\section{Intervention adherence}

Of the participants randomized to intervention, 12 of 13 women attended $100 \%$ of the 11 weight loss counseling sessions; one woman missed one session. A total of 142 weight loss counseling sessions occurred across the 13 women, with 89 (62.7\%) sessions occurring in-person and 53 (37.3\%) sessions occurring via telephone.

\section{Changes in body composition}

At 6 months, there was a $6.7 \%$ weight loss in the intervention group versus a $2.0 \%$ weight gain in the usual care group ( $p<$ 0.0001 ) (Table 3). Percent body fat decreased by $4.9 \%$ in the intervention group and increased by $5.1 \%$ in the usual care group. The group difference was significant $(p=0.008)$.

\section{Changes in serum biomarkers}

Post-intervention, leptin significantly decreased by $27.0 \%$ in the intervention group compared to an increase by $7.0 \%$ in the usual care group ( $p=0.03$ ) (Table 3 ). Adiponectin significantly increased by $25.5 \%$ in the exercise group compared to a decrease by $3.2 \%$ in the usual care group $(p=0.03)$. No other biomarkers changed differently by arms $(p>0.05)$.

\section{Changes in breast tissue biomarkers}

At 6 months, Ki67 increased $17.9 \%$ in the weight loss group and $25.8 \%$ in the usual care group $(p=0.51)$ (Table 3$)$. Insulin receptor expression increased $26.9 \%$ in the weight loss group and decreased $3.4 \%$ in the usual care group $(p=0.54)$. CD163 significantly decreased $15.9 \%$ in the weight loss group compared to a $34.3 \%$ increase in the usual care group $(p=0.04)$. There was no significant effect of the intervention on CD68 $(p=0.87)$.

\section{Baseline correlation of breast tissue biomarkers with serum blood markers and body composition}

At baseline, there were statistically significant inverse correlations between breast tissue levels of insulin receptor with both percent body fat $(r=-0.47, p=0.03)$ and serum insulin levels $(r=-0.45$, $p=0.04$ ) (Table 4). There was also a significant inverse correlation between serum insulin levels and CD68 $(r=-0.47, p=0.03)$.

Baseline to 6-month change in correlation of breast tissue biomarkers with serum blood markers and body composition At month 6 (Table 5), change in percent body fat was inversely associated with insulin receptor $(r=-0.42, p=0.05)$, and change in Ki67 breast tissue expression was borderline significantly inversely associated with a change in serum levels of insulin $(r=-0.42, p=0.06)$. Change in CD68 breast tissue expression was inversely associated with a change in serum levels of CRP ( $r=$ $-0.49, p=0.02$ ). Change in CD163 breast tissue expression was statistically significantly correlated with change in bone mineral density $(r=-0.42, p=0.049)$. 


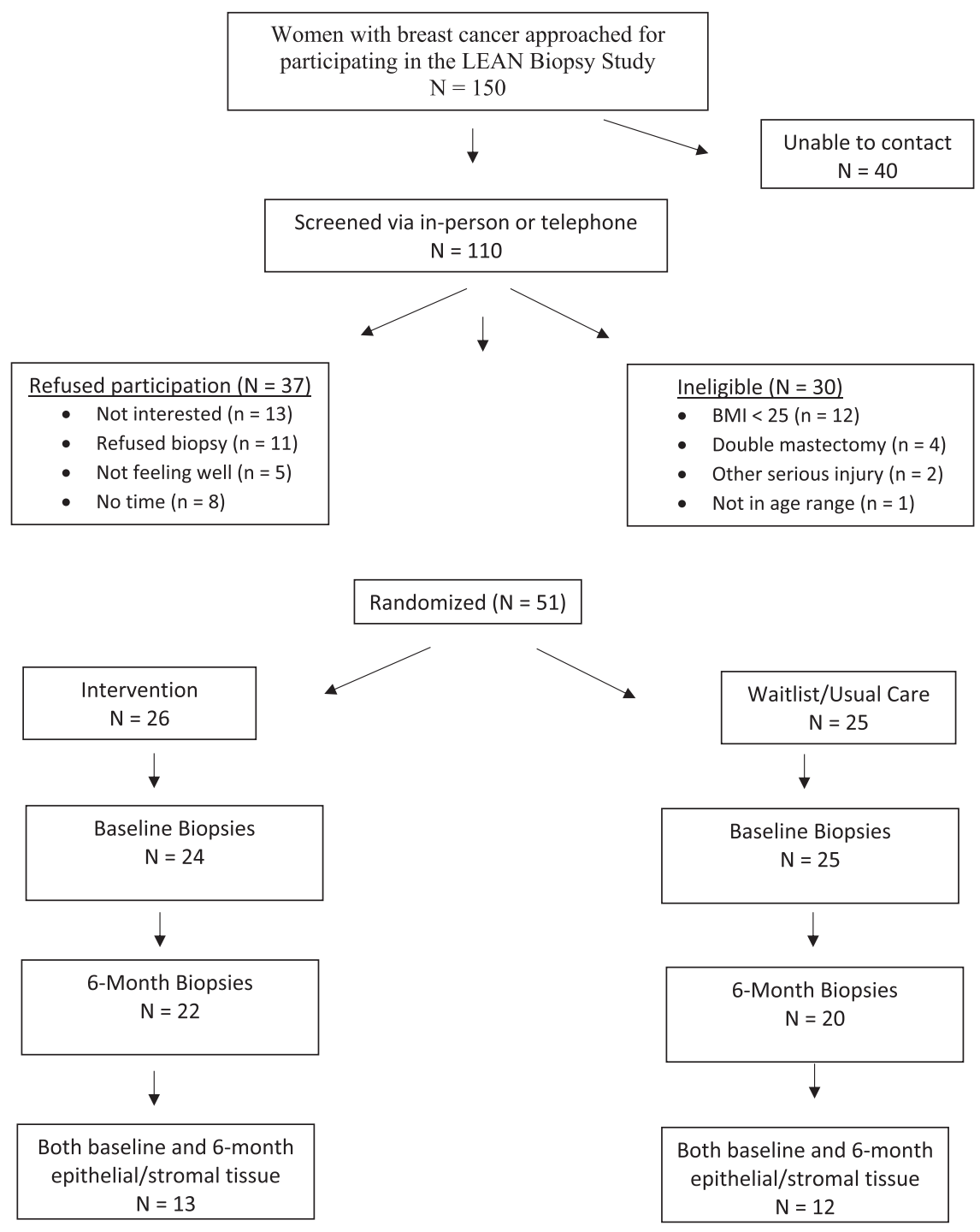

Fig. 1 Consort diagram for the LEAN trial. Recruitment of study participants.

\section{DISCUSSION}

A 6-month weight loss intervention in breast cancer survivors who completed chemotherapy and/or radiation therapy led to changes in body weight and fat, serum levels of leptin and adiponectin, and breast tissue levels of CD163.

Adherence to the intervention was high with 12 of 13 women attending $100 \%$ of the 11 weight loss counseling sessions. Furthermore, we demonstrated the feasibility of performing research biopsies of the breast in breast cancer survivors. Fiftyone $(46 \%)$ of the women approached to participate in the study agreed to undergo two biopsies; of these $100 \%$ of participants assigned to the intervention and $92 \%$ of participants assigned to the usual care, the group underwent both biopsies. We used a core needle biopsy procedure with a 14-gauge needle without ultrasound guidance which differs from the fine needle aspirate approach used in breast cancer prevention studies ${ }^{15}$. Due to the biopsying of normal tissue, the use of ultrasound guidance was not required thus further ensuring cost savings and preventing scheduling challenges required for ultrasound room reservations.

To our knowledge, our study is the first to examine the effect of a weight loss intervention on breast tissue changes in women with breast cancer. The Pre-Operative Health and Body (PreHAB) study tested an aerobic and resistance training intervention consisting of supervised aerobic exercise, followed by strength training, on women diagnosed with breast cancer $(n=49)$. Additional exercise prescription consisted of home-based aerobic exercise to achieve a total of $220 \mathrm{~min}$ of exercise per week in a mean of 29.3 days prior to breast surgery. The study demonstrated that the intervention, which increased exercise of the patients by 200 min per week from baseline, did not change Ki67 expression but significantly reduced leptin $(-12 \%)$ and upregulated cytokine-cytokine receptor interactions, NF-kB and chemokine signaling, and natural killer cell-mediated cell cytotoxicity ${ }^{16}$.

While both the LEAN and PreHAB studies did not report significant alterations in breast tissue following weight loss or exercise interventions, these two studies (a) demonstrated the feasibility of the collection of breast tissue and (b) laid the foundation for larger randomized controlled trials to assess lifestyle interventions on alterations in both breast tumor and healthy tissue. Other studies have examined the impact of the same in prostate tumor tissue in men with prostate cancer $^{10}$, in abdominal subcutaneous adipose tissue in postmenopausal women without a history of cancer ${ }^{8}$, and in colonic mucosal crypts of men and women without a history of cancer $^{11}$. We found that a weight loss intervention did not alter breast tissue expression of Ki67, IR, CD68; serum levels, however, were changed, with leptin and CRP decreasing, and adiponectin increasing in the presence of weight loss yet additional larger studies are warranted. 
Table 2. Baseline participant characteristics, means (SD) or $n(\%)$.

\begin{tabular}{|c|c|c|c|c|}
\hline & $\begin{array}{l}\text { Total } \\
N=25\end{array}$ & $\begin{array}{l}\text { Intervention } \\
N=13\end{array}$ & $\begin{array}{l}\text { Usual care } \\
N=12\end{array}$ & $P$ value \\
\hline Age (years) & $56.8(8.9)$ & $59.5(6.5)$ & $54.1(10.6)$ & 0.14 \\
\hline $\begin{array}{l}\text { Time from diagnosis } \\
\text { (years) }\end{array}$ & $3.3(3.8)$ & $2.8(2.4)$ & $3.8(4.9)$ & 0.51 \\
\hline Baseline weight $(\mathrm{kg})$ & $85.5(16.2)$ & $75.9(7.8)$ & $95.6(16.8)$ & 0.002 \\
\hline Baseline BMI & $32.8(6.0)$ & $29.5(3.6)$ & $36.4(6.1)$ & 0.002 \\
\hline $\mathrm{BMI} \geq 30 \mathrm{~kg} / \mathrm{m}^{2}$ & $15(60 \%)$ & $5(38 \%)$ & 10 (83\%) & 0.04 \\
\hline Race/Ethnicity & & & & 0.10 \\
\hline $\begin{array}{l}\text { Black or African- } \\
\text { American }\end{array}$ & $2(8 \%)$ & & $2(17 \%)$ & \\
\hline Non-Hispanic White & $22(88 \%)$ & $13(100 \%)$ & $9(75 \%)$ & \\
\hline Other & $1(4 \%)$ & & $1(4 \%)$ & \\
\hline Education & & & & 0.40 \\
\hline High school degree & $3(12 \%)$ & $1(8 \%)$ & $2(17 \%)$ & \\
\hline $\begin{array}{l}\text { Some } \\
\text { college degree }\end{array}$ & $8(32 \%)$ & $3(23 \%)$ & $5(42 \%)$ & \\
\hline College degree & $6(24 \%)$ & $5(38 \%)$ & $1(8 \%)$ & \\
\hline Graduate degree & $8(32 \%)$ & $4(31 \%)$ & $3(23 \%)$ & \\
\hline Postmenopausal & $22(88 \%)$ & 12 (92\%) & $10(83 \%)$ & 0.49 \\
\hline Cancer stage & & & & 0.76 \\
\hline DCIS & $4(15 \%)$ & $1(8 \%)$ & $3(25 \%)$ & \\
\hline Stage I & $13(52 \%)$ & $8(62 \%)$ & $5(42 \%)$ & \\
\hline Stage II & $6(24 \%)$ & $3(23 \%)$ & $3(23 \%)$ & \\
\hline Stage III & $2(8 \%)$ & $1(8 \%)$ & $1(8 \%)$ & \\
\hline $\begin{array}{l}\text { Current endocrine } \\
\text { therapy }\end{array}$ & & & & 0.88 \\
\hline $\begin{array}{l}\text { Aromatase } \\
\text { inhibitors }\end{array}$ & $10(42 \%)$ & $6(46 \%)$ & $4(36 \%)$ & \\
\hline Tamoxifen & $6(24 \%)$ & $3(23 \%)$ & $3(27 \%)$ & \\
\hline None & $8(33 \%)$ & $4(31 \%)$ & $4(36 \%)$ & \\
\hline $\begin{array}{l}\text { Prior adjuvant } \\
\text { treatment }\end{array}$ & & & & 0.47 \\
\hline None & $1(4 \%)$ & 0 & $1(8 \%)$ & \\
\hline Radiation only & $13(52 \%)$ & $8(62 \%)$ & $5(42 \%)$ & \\
\hline Chemotherapy only & $1(4 \%)$ & 0 & $1(8 \%)$ & \\
\hline $\begin{array}{l}\text { Radiation and } \\
\text { chemotherapy }\end{array}$ & 10 (38\%) & $5(38 \%)$ & $5(42 \%)$ & \\
\hline
\end{tabular}

Various associations of body composition and serum and tissue biomarkers indicate the necessity of future explorations in this area. In our study, we observed body fat was inversely related to IR gene expression in the breast tissue despite a nonsignificant increase in IR gene expression in the intervention group. This suggests that with a larger sample statistical significance may have been attained. Goodwin et al. (2007) had previously reported higher expression of IR in breast tumor tissue to be independently and significantly associated with more favorable clinical outcomes including low tumor grade, lymph node negativity, progesterone positivity, and improved breast cancer and overall survival ${ }^{17}$. In addition, we found an inverse association between CRP and CD68/CD163. Given that CD68 and CD163 are macrophage markers of the M1 and M2 phenotype, and CRP is produced by macrophages, it is plausible that our intervention impacted the ratio of $M 1 / M 2$. We had previously found that aerobic and resistance exercise intervention positively alters $\mathrm{M} 1 / \mathrm{M} 2$ macrophage phenotype within subcutaneous abdominal adipose tissue of breast cancer survivors ${ }^{18}$.
It is important to recognize our examination of healthy breast tissue rather than breast tumor tissue, as women who participated in this trial had completed therapy and were free of active disease. It is known that benign breast tissue is susceptible to biomarker changes due to modifications in body weight. Fabian et al. ${ }^{19}$, for example, reported favorable changes in tissue levels of Ki-67 (among 15 women with measurable Ki-67), adiponectin:leptin, cyclin B1, phosphorylated retinoblastoma, and ribosomal $\mathrm{S} 6$ proteins in breast tissue of postmenopausal overweight or obese women who had lost weight over a 6 -month period ${ }^{19}$. It is plausible that post-treatment weight loss interventions that alter breast tissue biology elicit a similar protective response to breast cancer recurrence.

Limitations of our study must be acknowledged. Overall, $46 \%$ of women screened for the study consented to the two biopsies, implying feasibility. Our $46 \%$ enrollment rate $(51 / 110)$ is higher than we hypothesized and higher than other randomized trials of lifestyle behaviors in healthy women and women with breast cancer (e.g., $2.2 \%$ in an exercise trial of postmenopausal women ${ }^{20}$; $11.9 \%$ in our exercise trial in women with breast cancer $^{21}$ ). The $50 \%$ (i.e., 25/51) of breast tissue yielding epithelial tissue is lower than we hypothesized yet understandable given biopsies were performed without ultrasound guidance. Using ultrasound may have increased the number of samples with epithelial tissue ${ }^{22}$ yet would also increase the cost of the study and also add scheduling challenges. Other study limitations are that imbalance in obesity at baseline across groups, lack of adjustment for multiple comparisons, our final sample size was underpowered for the efficacy outcomes, and in theory, biopsy specimens obtained from a single site may not be indicative of whole breast tissue alterations.

In conclusion, our weight loss trial led to favorable changes in certain metabolic and inflammatory biomarkers and body composition. This first-of-its-kind study supports the feasibility of breast tissue biopsies obtained to test the impact of lifestyle interventions on tissue biology among breast cancer survivors.

\section{METHODS}

The LEAN study (NCT02110641) was a randomized trial comparing a weight loss intervention to usual care. All measures were collected at baseline and 6 months. All procedures, including written informed consent, were approved by the Yale School of Medicine Human Investigation Committee.

\section{Participants and recruitment}

We recruited a new cohort of women treated for breast cancer into the LEAN biopsy study. Women were recruited between 1 February 2014 and 1 January 2016 from Smilow Cancer Hospital medical oncology clinics at Yale, self-referral via study brochures in the Breast Center at Smilow Cancer Hospital, and the Yale Cancer Center Survivorship Clinic. Eligible participants were women diagnosed with breast cancer, with a $\mathrm{BMI} \geq$ $25.0 \mathrm{~kg} / \mathrm{m}^{2}$, who had completed chemotherapy and/or radiation therapy. Women had to be physically able to exercise, give informed consent, be accessible by telephone, and be able to communicate in English.

\section{Breast tissue biopsies}

Core needle biopsies using a 14-gauge needle were taken from the unaffected breast following standard guidelines and protocols. Three cores were taken: one core was snap frozen, one core placed in $10 \%$ neutral buffered formalin, and one core placed in RNAlater ${ }^{\mathrm{TM}}$. Both frozen tissue and tissues placed in RNAlater ${ }^{\mathrm{TM}}$ solution were stored at a lab of Yale Tissue Pathology Services (YPTS) until further analysis. Formalin-fixed, paraffin-embedded biopsy specimens collected in this study were analyzed for KI67, Insulin receptor, CD68, and CD163.

\section{Ki67 and insulin receptor quantitative immunofluorescence (QIF) staining}

Fresh cuts of LEAN study whole tissue slides were deparaffinized and rehydrated before undergoing antigen retrieval using citrate buffer $(\mathrm{pH}=$ 6) for $20 \mathrm{~min}$ at $97^{\circ} \mathrm{C}$ (PT module, Lab Vision, Thermo Fisher Scientific, Waltham, MA). Slides were incubated with a solution of $0.3 \%$ hydrogen 
Table 3. Baseline to 6-month change in breast tissue biomarkers, serum biomarkers, and weight compositions controlling for baseline values and baseline BMI except for BMI itself.

\begin{tabular}{|c|c|c|c|}
\hline Variables & Intervention, mean (SD), $n=13$ & Control, mean (SD), $n=12$ & $P$ value \\
\hline Baseline Ki67 (AQUA score) $^{\mathrm{a}}$ & $438.1(225.9)$ & $434.2(206.2)$ & 0.97 \\
\hline Baseline insulin receptor & $3322(2920)$ & $2944(1567)$ & 0.70 \\
\hline 6-month change in insulin receptor & $893.4(2514)$ & $-99.7(2329)$ & 0.54 \\
\hline Baseline CD163 & $5277(2416)$ & $3882(1830)$ & 0.14 \\
\hline 6-month change in CD163 & $-839(3905)$ & $1331(3666)$ & 0.04 \\
\hline \multicolumn{4}{|l|}{ Serum biomarkers } \\
\hline Baseline Leptin, ng/ml & $31.8(14.4)$ & $55.0(34.0)$ & 0.045 \\
\hline 6-month change in Leptin & $-8.6(11.6)$ & $3.9(17.6)$ & 0.03 \\
\hline Baseline IGF1, ng/ml & $88.30(21.15)$ & $101.0(33.68)$ & 0.27 \\
\hline 6-month IGF1 & $7.01(29.22)$ & $0.12(17.61)$ & 0.52 \\
\hline Baseline Insulin, $\mu \mathrm{U} / \mathrm{ml}$ & $7.96(5.41)$ & $11.12(5.68)$ & 0.17 \\
\hline 6-month change insulin & $-1.47(2.97)$ & $-0.12(3.53)$ & 0.53 \\
\hline Baseline IL-6, pg/ml & $2.61(1.30)$ & $4.01(1.70)$ & 0.03 \\
\hline 6-month change IL-6 & $0.19(2.19)$ & $-0.29(0.98)$ & 0.78 \\
\hline Baseline TNFa, pg/ml & $0.68(0.28)$ & $0.75(0.30)$ & 0.56 \\
\hline 6-month change TNFa & $-0.03(0.32)$ & $0.02(0.27)$ & 0.16 \\
\hline \multicolumn{4}{|l|}{ Body composition } \\
\hline Baseline BMI $\left(\mathrm{kg} / \mathrm{m}^{2}\right)$ & $29.54(3.59)$ & $36.38(6.06)$ & 0.002 \\
\hline Baseline weight, $\mathrm{kg}$ & $75.85(7.83)$ & $95.64(16.84)$ & 0.002 \\
\hline 6-month change in weight, kg & $-5.05(3.24)$ & $1.88(2.37)$ & $<0.000$ \\
\hline
\end{tabular}

peroxide in methanol to inactivate endogenous peroxidase for $30 \mathrm{~min}$, followed: $30 \mathrm{~min}$ of incubation with $0.3 \%$ bovine serum albumin with $0.05 \%$ Tween-20 blocking solution, incubation with a cocktail of Ki67 (mouse IgG1, Clone MIB-1, DaKo Corp, Carpinteria, CA) at $0.46 \mu \mathrm{g} / \mathrm{ml}$ and a wide-spectrum rabbit anti-cow cytokeratin antibody (Z0622; Dako Corp, Carpinteria, CA), followed by a $1-\mathrm{h}$ incubation at room temperature with Alexa 546-conjugated goat anti-rabbit secondary antibody (A11010; Molecular Probes, Eugene, OR). Cyanine 5 (Cy5) directly conjugated to tyramide (FP1117; Perkin-Elmer, Boston, MA) at a 1:50 dilution was used as the fluorescent chromogen for Ki67 detection. Insulin receptor staining followed the same protocol with the primary antibody (Insulin receptor beta subunit monoclonal antibody, clone CT-3, mouse IgG1, Enzo Life Sciences, Farmingdale, NY) at $2 \mu \mathrm{g} / \mathrm{ml}$.

\section{CD68 and CD163 multiplexed QIF staining}

Fresh cuts of LEAN study whole tissue slides were deparaffinized and rehydrated before undergoing antigen retrieval using an EDTA buffer $(\mathrm{pH}=8)$ for $20 \mathrm{~min}$ at $97^{\circ} \mathrm{C}$ (PT module, Lab Vision, Thermo Fisher Scientific, Waltham, MA, USA). Next, we incubated the slides with a solution of $0.3 \%$ hydrogen peroxide in methanol to inactivate endogenous peroxidase for $30 \mathrm{~min}$, followed by another $30 \mathrm{~min}$ of incubation with $0.3 \%$ bovine serum albumin with $0.05 \%$ Tween-20 blocking solution. Fluorescent staining for pancytokeratin, CD68, and CD163, biomarkers of inflammation, was performed by using a sequential multiplexed protocol with different isotype-specific primary antibodies. Antibodies against these targets were used to detect epithelial tumor cells (Anti-wide spectrum Cytokeratin antibody, Abcam, Cambridge, $M A)$, all macrophages (CD68, mouse monoclonal lgG3, clone PG-M1, Dako Corp, $0.15 \mu \mathrm{g} / \mathrm{mL}$ ) and M2-class macrophages (CD163, mouse monoclonal $\operatorname{lgG1}$, clone $10 \mathrm{D6}$ from Leica, Wetzlar, Germany, $0.006 \mu \mathrm{g} / \mathrm{mL}$ ).

\section{Fluorescence signal quantification}

We used the AQUA method of QIF (Navigate Biopharma), to quantify the fluorescence signal of Ki67, IR, CD68, CD163 that were measured within whole tissue compartments. QIF scores were calculated by dividing the target pixel intensity by the area of the compartment of interest ${ }^{23}$ and then normalized to the exposure time and bit depth at which the images were captured. 
Table 4. Baseline correlations for body composition, serum, and breast tissue biomarkers, $n=25$ ( $r, p$-value).

\begin{tabular}{lrrrr}
\hline & \multicolumn{1}{c}{ Ki67 } & \multicolumn{1}{l}{ IR } & CD68 & CD163 \\
\hline BMI $\left(\mathrm{kg} / \mathrm{m}^{2}\right)$ & 0.12 & -0.38 & -0.09 & -0.06 \\
& 0.60 & 0.09 & 0.71 & 0.80 \\
BMD $\left(\mathrm{g} / \mathrm{cm}^{-2}\right)$ & -0.01 & 0.09 & 0.10 & -0.55 \\
& 0.97 & 0.70 & 0.67 & 0.01 \\
Lean body mass $(\mathrm{kg})$ & -0.12 & -0.23 & -0.17 & -0.31 \\
& 0.61 & 0.31 & 0.46 & 0.17 \\
\% Body fat & 0.08 & -0.47 & 0.05 & -0.08 \\
& 0.72 & 0.03 & 0.83 & 0.74 \\
Leptin (pg/ml) & 0.17 & -0.33 & -0.21 & -0.01 \\
& 0.48 & 0.14 & 0.34 & 0.95 \\
CRP (ng/ml) & 0.03 & 0.04 & 0.18 & 0.12 \\
& 0.88 & 0.87 & 0.43 & 0.60 \\
Adiponectin (ng/ml) & 0.09 & -0.16 & -0.22 & 0.30 \\
& 0.71 & 0.47 & 0.33 & 0.17 \\
IGF-I (ng/ml) & 0.07 & 0.17 & 0.16 & 0.12 \\
& 0.76 & 0.46 & 0.48 & 0.58 \\
Insulin $(\mu \mathrm{U} / \mathrm{ml})$ & 0.36 & -0.45 & -0.47 & 0.04 \\
& 0.12 & 0.04 & 0.03 & 0.88 \\
IL-6 (pg/ml) & -0.13 & 0.11 & 0.03 & -0.09 \\
& 0.58 & 0.65 & 0.88 & 0.69 \\
TNFa (pg/ml) & -0.24 & 0.14 & 0.31 & -0.26 \\
& 0.31 & 0.54 & 0.16 & 0.23 \\
\hline
\end{tabular}

Table 5. Correlations for 6-month changes in body composition, serum, and breast tissue biomarkers, $n=25$ ( $r, p$-value).

\begin{tabular}{lrrrr}
\hline & \multicolumn{1}{c}{ Ki67 } & \multicolumn{1}{c}{ IR } & CD68 & cd163 \\
\hline Leptin & -0.32 & -0.13 & 0.120 & 0.09 \\
& 0.17 & 0.59 & 0.37 & 0.70 \\
CRP & 0.18 & -0.17 & -0.49 & -0.08 \\
& 0.45 & 0.45 & 0.02 & 0.74 \\
Adiponectin & 0.05 & 0.14 & -0.27 & -0.29 \\
& 0.84 & 0.55 & 0.22 & 0.19 \\
IGFI & -0.29 & 0.03 & -0.17 & -0.32 \\
& 0.22 & 0.90 & 0.44 & 0.14 \\
Insulin & -0.42 & 0.09 & -0.08 & 0.25 \\
& 0.06 & 0.70 & 0.71 & 0.27 \\
IL6 & -0.00 & -0.08 & -0.32 & -0.16 \\
& 0.10 & 0.73 & 0.14 & 0.47 \\
TNFa & -0.23 & -0.17 & -0.22 & -0.30 \\
& 0.34 & 0.46 & 0.33 & 0.18 \\
LBM & -0.19 & 0.09 & 0.21 & 0.11 \\
& 0.43 & 0.70 & 0.35 & 0.61 \\
\% Body fat & 0.06 & -0.42 & -0.02 & 0.36 \\
& 0.79 & 0.05 & 0.93 & 0.10 \\
BMD & 0.34 & 0.02 & -0.34 & -0.42 \\
& 0.14 & 0.94 & 0.12 & 0.05 \\
BMI & -0.03 & -0.38 & 0.22 & 0.38 \\
& 0.90 & 0.09 & 0.33 & 0.08 \\
\hline
\end{tabular}

\section{Anthropometrics and body composition}

Height was measured using a stadiometer. Weight was measured while participants were wearing light indoor clothing, without shoes. Dualenergy X-ray absorptiometry scans were performed to assess body fat, lean body mass, and bone mineral density using a Hologic 4500 scanner.

\section{Blood draw and serum biomarkers}

A fasting ( $\geq 12 \mathrm{~h}$ ) blood draw was performed and serum samples were stored at $-80^{\circ} \mathrm{C}$ until assayed. Serum concentrations of insulin, leptin, and adiponectin were measured using radioimmunoassay kits; IL- 6 and TNF- $a$ were measured using high-sensitivity enzyme-linked immunoabsorbent assay kits; and C-reactive protein (CRP) was measured using an automated chemistry analyzer.

\section{Physical activity and dietary intake}

At baseline and 6 months, participants completed the Modifiable Physical Activity Questionnaire (MPAQ) administered by an interviewer. The past 6 months of physical activity was assessed including frequency, duration, and type using a validated physical activity questionnaire ${ }^{24,25}$. Past month dietary intake was assessed at baseline and 6 months by a 120-item food frequency questionnaire developed for the Women's Health Initiative Study ${ }^{26}$.

\section{Weight loss intervention}

The weight loss intervention was adapted from the Diabetes Prevention Program ${ }^{27}$ with updates using the 2010 US Dietary Guidelines ${ }^{28}$ and adapted to breast cancer survivors using the American Institute for Cancer Research/World Cancer Research Fund and American Cancer Society nutrition and physical activity guidelines ${ }^{3}$. The intervention included a combination of reduced caloric intake, increased physical activity, and behavioral therapy. Participants received individualized counseling sessions once per week (month 1), then every two weeks (months 2 and 3), and once per month (months, 4, 5, and 6). Each of the 11 sessions was $30 \mathrm{~min}$ in duration, and represented a core curriculum with specific information about nutrition, exercise, and behavior strategies from the 11chapter LEAN book developed for the LEAN Study ${ }^{14}$. Given our previous study comparing in-person and telephone weight loss counseling to usual care found no difference in weight loss via in-person or telephone ${ }^{12}$, participants were able to choose if a session occurred in-person or via telephone. Participants recorded all food and beverage intake, minutes of physical activity and pedometer steps in the LEAN Journal on a daily basis. Participants were provided with a scale (HoMedics) to weigh themselves once per week, and recorded their weight in the LEAN journal.

Participants were instructed to reduce energy intake to the range of 1200 to $2000 \mathrm{kcal} /$ day based upon baseline weight and to incur in an energy deficit of $500 \mathrm{kcal} /$ day. The dietary fat goal was $<25 \%$ of total energy intake. The home-based physical activity program included a goal of $150 \mathrm{~min}$ per week of moderate-intensity activity, such as brisk walking. Women were given a pedometer and coached to increase their number of steps to 10,000 per day. Reducing sedentary behaviors was encouraged through activities of daily living.

\section{Usual care group}

The usual care group was provided with the American Institute for Cancer Research nutrition and physical activity brochures and referred to the Yale Cancer Center Survivorship Clinic which offers a two-session weight management program. At the completion of the study, usual care participants were offered the entire LEAN program including the 11 counseling sessions, LEAN book, and LEAN journal.

\section{Statistical analyses}

Demographics and other baseline characteristics were summarized as means and standard deviations for continuous variables or frequencies and percentage for categorical variables. The baseline characteristics were compared using $t$ test or Chi-square test as appropriate. Baseline body composition variables and biomarkers were compared using $t$ test, while 6-month changes of body composition variables and biomarkers were compared between groups using the analysis of covariance (ANCOVA) method with covariate adjustment for baseline value and baseline BMI. Spearman correlation analysis was used to explore the relationship between tissue and serum biomarkers and body composition outcomes at baseline as well as 6-month change from baseline. SAS 9.4 (Cary, NC) 
was used for all statistical analyses. Statistical significance was set as $p<$ 0.05 , two-sided. Since all the analyses were exploratory, raw $p$ values were reported without adjusting for multiple comparisons ${ }^{29}$.

\section{Reporting summary}

Further information on research design is available in the Nature Research Reporting Summary linked to this article.

\section{DATA AVAILABILITY}

The data generated or analyzed during and/or analyzed during the current study are available from the corresponding author on reasonable request.

Received: 17 August 2021; Accepted: 1 February 2022;

Published online: 07 March 2022

\section{REFERENCES}

1. Lauby-Secretan, B. et al. Body fatness and cancer-viewpoint of the IARC working group. N. Engl. J. Med. 375, 794-798 (2016).

2. Chan, D. S. et al. Body mass index and survival in women with breast cancersystematic literature review and meta-analysis of 82 follow-up studies. Ann Oncol. 25, 1901-1914 (2014).

3. Rock, C. L. et al. Nutrition and physical activity guidelines for cancer survivors. $C A$ Cancer J. Clin. 62, 243-274 (2012)

4. Jiralerspong, S. et al. Obesity, diabetes, and survival outcomes in a large cohort of early-stage breast cancer patients. Ann. Oncol. 24, 2506-2514 (2013).

5. Mason, C. et al. Long-term physical activity trends in breast cancer survivors. Cancer Epidemiol. Biomark. Prev. 22, 1153-1161 (2013).

6. Calle, E. E. \& Kaaks, R. Overweight, obesity and cancer: epidemiological evidence and proposed mechanisms. Nat. Rev. Cancer 4, 579-591 (2004).

7. Bianchini, F., Kaaks, R. \& Vainio, H. Overweight, obesity, and cancer risk. Lancet Oncol. 3, 565-574 (2002).

8. Campbell, K. L. et al. Gene expression changes in adipose tissue with diet- and/or exercise-induced weight loss. Cancer Prev. Res (Philos.) 6, 217-231 (2013).

9. Ligibel, J. A. et al. Impact of a pre-operative exercise intervention on breast cancer proliferation and gene expression: results from the Pre-Operative Health and Body (PreHAB) Study. Clin. Cancer Res. 25, 5398-5406 (2019).

10. Demark-Wahnefried, W. et al. Presurgical weight loss affects tumour traits and circulating biomarkers in men with prostate cancer. Br. J. Cancer 117, 1303-1313 (2017).

11. McTiernan, A. et al. Effect of a 12-month exercise intervention on patterns of cellular proliferation in colonic crypts: a randomized controlled trial. Cancer Epidemiol. Biomark. Prev. 15, 1588-1597 (2006).

12. Santa-Maria, C. A. et al. The effects of a remote-based weight loss program on adipocytokines, metabolic markers, and telomere length in breast cancer survivors: the POWER-Remote Trial. Clin. Cancer Res. 26, 3024-3034 (2020).

13. Sauter, E. R. Reliable biomarkers to identify new and recurrent cancer. Eur. J. Breast Health 13, 162-167 (2017).

14. Harrigan, M. et al. Randomized trial comparing telephone versus in-person weight loss counseling on body composition and circulating biomarkers in women treated for breast cancer: the Lifestyle, Exercise, and Nutrition (LEAN) Study. J. Clin. Oncol. 34, 669-676 (2016).

15. Fabian, C. J. et al. Short-term breast cancer prediction by random periareolar fineneedle aspiration cytology and the Gail risk model. J. Natl Cancer Inst. 92 1217-1227 (2000).

16. Ligibel, J. A. et al. Impact of a pre-operative exercise intervention on breast cancer proliferation and gene expression: results from the Pre-Operative Health and Body (PreHAB) Study. Clin. Cancer Res 25, 5398-5406 (2019).

17. Mulligan, A. M., O'Malley, F. P., Ennis, M., Fantus, I. G. \& Goodwin, P. J. Insulin receptor is an independent predictor of a favorable outcome in early stage breast cancer. Breast Cancer Res. Treat. 106, 39-47 (2007).

18. Dieli-Conwright, C. M. et al. Adipose tissue inflammation in breast cancer survivors: effects of a 16-week combined aerobic and resistance exercise training intervention. Breast Cancer Res. Treat. 168, 147-157 (2018).

19. Fabian, C. J. et al. Favorable modulation of benign breast tissue and serum risk biomarkers is associated with $>10 \%$ weight loss in postmenopausal women. Breast Cancer Res. Treat. 142, 119-132 (2013).
20. McTiernan, A. et al. Effect of exercise on serum estrogens in postmenopausal women: a 12-month randomized clinical trial. Cancer Res. 64, 2923-2928 (2004).

21. Irwin, M. L. et al. Randomized exercise trial of aromatase inhibitor-induced arthralgia in breast cancer survivors. J. Clin. Oncol. 33, 1104-1111 (2015).

22. Kim, W. H. et al. Ultrasonographic assessment of breast density. Breast Cancer Res. Treat. 138, 851-859 (2013).

23. Camp, R. L., Chung, G. G. \& Rimm, D. L. Automated subcellular localization and quantification of protein expression in tissue microarrays. Nat. Med. 8, 1323-1327 (2002).

24. Kriska, A. M. C. \& C, J. A collection of physical activity questionnaires. Med Sci. Sports Exer. 29, S79-S82 (1997).

25. Kriska, A. M. et al. Development of questionnaire to examine relationship of physical activity and diabetes in Pima Indians. Diabetes Care 13, 401-411 (1990).

26. Patterson, R. E. et al. Measurement characteristics of the Women's Health Initiative food frequency questionnaire. Ann. Epidemiol. 9, 178-187 (1999).

27. Knowler, W. C. et al. Reduction in the incidence of type 2 diabetes with lifestyle intervention or metformin. N. Engl. J. Med. 346, 393-403 (2002).

28. Bakker, M. F. et al. Plasma carotenoids, vitamin C, tocopherols, and retinol and the risk of breast cancer in the European Prospective Investigation into Cancer and Nutrition cohort. Am. J. Clin. Nutr. 103, 454-464 (2016).

29. Bender, R. \& Lange, S. Adjusting for multiple testing-when and how? J. Clin. Epidemiol. 54, 343-349 (2001).

\section{ACKNOWLEDGEMENTS}

TREC Training Workshop R25CA203650 (PI: Melinda Irwin).

\section{AUTHOR CONTRIBUTIONS}

Study design and execution: M.I., B.C., A.C., Y.B., F.L., D.R., L.P., L.L., and T.S.; Data analysis: F.L.; manuscript writing and revisions: all authors.

\section{COMPETING INTERESTS}

The authors declare no competing interests.

\section{ADDITIONAL INFORMATION}

Supplementary information The online version contains supplementary materia available at https://doi.org/10.1038/s41523-022-00396-z.

Correspondence and requests for materials should be addressed to Christina $M$. Dieli-Conwright.

Reprints and permission information is available at http://www.nature.com/ reprints

Publisher's note Springer Nature remains neutral with regard to jurisdictional claims in published maps and institutional affiliations.

Open Access This article is licensed under a Creative Commons Attribution 4.0 International License, which permits use, sharing, adaptation, distribution and reproduction in any medium or format, as long as you give appropriate credit to the original author(s) and the source, provide a link to the Creative Commons license, and indicate if changes were made. The images or other third party material in this article are included in the article's Creative Commons license, unless indicated otherwise in a credit line to the material. If material is not included in the article's Creative Commons license and your intended use is not permitted by statutory regulation or exceeds the permitted use, you will need to obtain permission directly from the copyright holder. To view a copy of this license, visit http://creativecommons. org/licenses/by/4.0/.

(c) The Author(s) 2022 